\title{
Archéopages
}

Archéopages

Archéologie et société

37 | 04/2013

Jardins

\section{Nouvelle mention du Quoit Brooch Style en Bretagne}

\author{
Françoise Labaune-Jean
}

\section{(2) OpenEdition}

1 Journals

Édition électronique

URL : https://journals.openedition.org/archeopages/366

DOI : 10.4000/archeopages.366

ISSN : 2269-9872

\section{Éditeur}

INRAP - Institut national de recherches archéologiques préventives

\section{Édition imprimée}

Date de publication : 1 février 2014

Pagination : 86-87

ISSN : 1622-8545

\section{Référence électronique}

Françoise Labaune-Jean, « Nouvelle mention du Quoit Brooch Style en Bretagne », Archéopages [En

ligne], 37 | 04/2013, mis en ligne le 01 avril 2015, consulté le 21 janvier 2022. URL : http://

journals.openedition.org/archeopages/366 ; DOI : https://doi.org/10.4000/archeopages.366 
Période

Haut Moyen Âge

\section{Nouvelle mention du Quoit Brooch Style en Bretagne}

Françoise Labaune-Jean

Inrap, UMR 6566 CreAAH, étude instrumentum

Site

Hôtel-Dieu, rue de la Cochardière Rennes

Ille-et-Vilaine

Date

Fin janvier-début mars 2012 Superficie

2,3 ha

\section{Équipe}

Responsable d'opération Dominique Pouille, Inrap Consolidation-restauration Alejandra Balboa-Garcia et Marina Biron, Inrap
Les sondages de diagnostic, menés fin 2011 sur l'emprise est non bâtie de l'ancien hôtel-Dieu de Rennes, ont permis de confirmer la présence d'un grand pôle à usage funéraire. Déjà perçu à deux reprises lors d'interventions voisines, dans les années 1970 et en 2001 (Bousquet, 1971 ; Pouille, 2001), ce secteur se situe en limite nord de

l'agglomération antique de Condate, dans un maillage urbain moins dense ayant déjà livré quelques sépultures du Bas-Empire. Cette nouvelle intervention montre, pour la première fois, que cet usage funéraire persiste également au cours du haut Moyen Âge, à proximité de l'église SaintMartin dont l'emplacement exact reste encore à préciser.

Bien que découvert dans les terres de surface, l'objet présenté ici peut être rattaché de manière quasi certaine à une inhumation de femme adulte. $\mathrm{La}$ fibule se présente sous la forme d'une plaque de métal découpée en forme d'oméga et dotée en surface d'un anneau fin sur lequel coulisse une pointe effilée [ill. 1]. Elle entre dans le groupe des fibules penannulaires, même si ce type à corps plat est moins fréquent que la variante épaisse (Suzuki, 200o). L'objet mesure 3,65 par $3,5 \mathrm{~cm}$, pour une épaisseur maximale de $0,3 \mathrm{~cm}$ et un poids de 7,31 g.

Après nettoyage et consolidation, cette fibule en argent a révélé une riche décoration gravée, dont la majeure partie se trouve sur le corps proprement dit. L'espace central de la plaque est gravé d'une figure de personnage à tête triangulaire, surmontée d'un motif courbe terminé en cornes. Les yeux en amande sont reliés par un nez en $U$. Le pourtour du visage est doublé de fines incisions évoquant une barbe régulière. On les retrouve également au-dessus de la tête pour la chevelure [ill. 2.a].
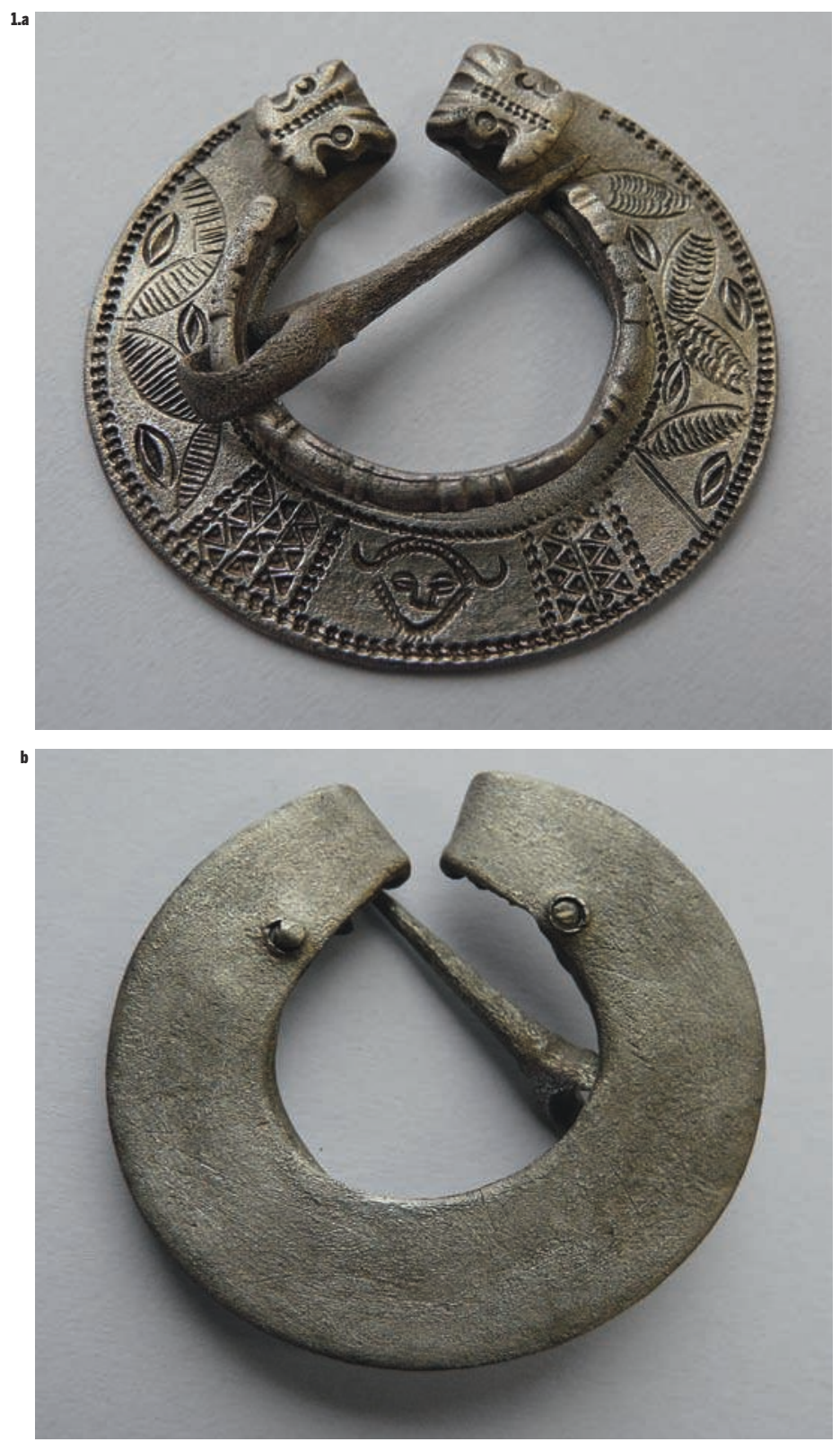

1 Vue de face (a) et

du revers (b) de la fibule

$\rightleftharpoons$

après nettoyage-

consolidation.

De part et d'autre du visage, un bandeau large se compose, respectivement à gauche et à droite, de deux ou trois lignes verticales à base de six triangles estampés placés en quinconce. Les bordures externes du bandeau sont faites de petits motifs également estampés en forme de $S$ épais, donnant une impression de corde tressée. Cet élément se retrouve de manière plus espacée pour la ligne longeant tout le bord externe de la fibule [ill. 2.b].

Sur la section de gauche, deux demicercles incisés sont accolés et tracés à partir du bord externe de la pièce.

Deux arcs sécants de même diamètre sont disposés à l'intérieur de chacun d'eux de manière à former deux feuilles fuselées dont la surface est hachurée à l'oblique. Les arcs et demi-cercles tracés au compas montrent de légers décalages ou débordements. Les interstices laissés libres à l'intérieur et entre eux sont occupés par trois petits motifs en navette à fente centrale. Sur la section de droite, après un petit espace vide, on retrouve le même dispositif mais avec une orientation inversée des demi-cercles vers le bord interne et un décor de double ligne de petites incisions courbes dans les segments. La ligne de bordure a été réalisée dans un second temps de manière à masquer en partie les trous de compas de cercles [ill. 2.c]. 

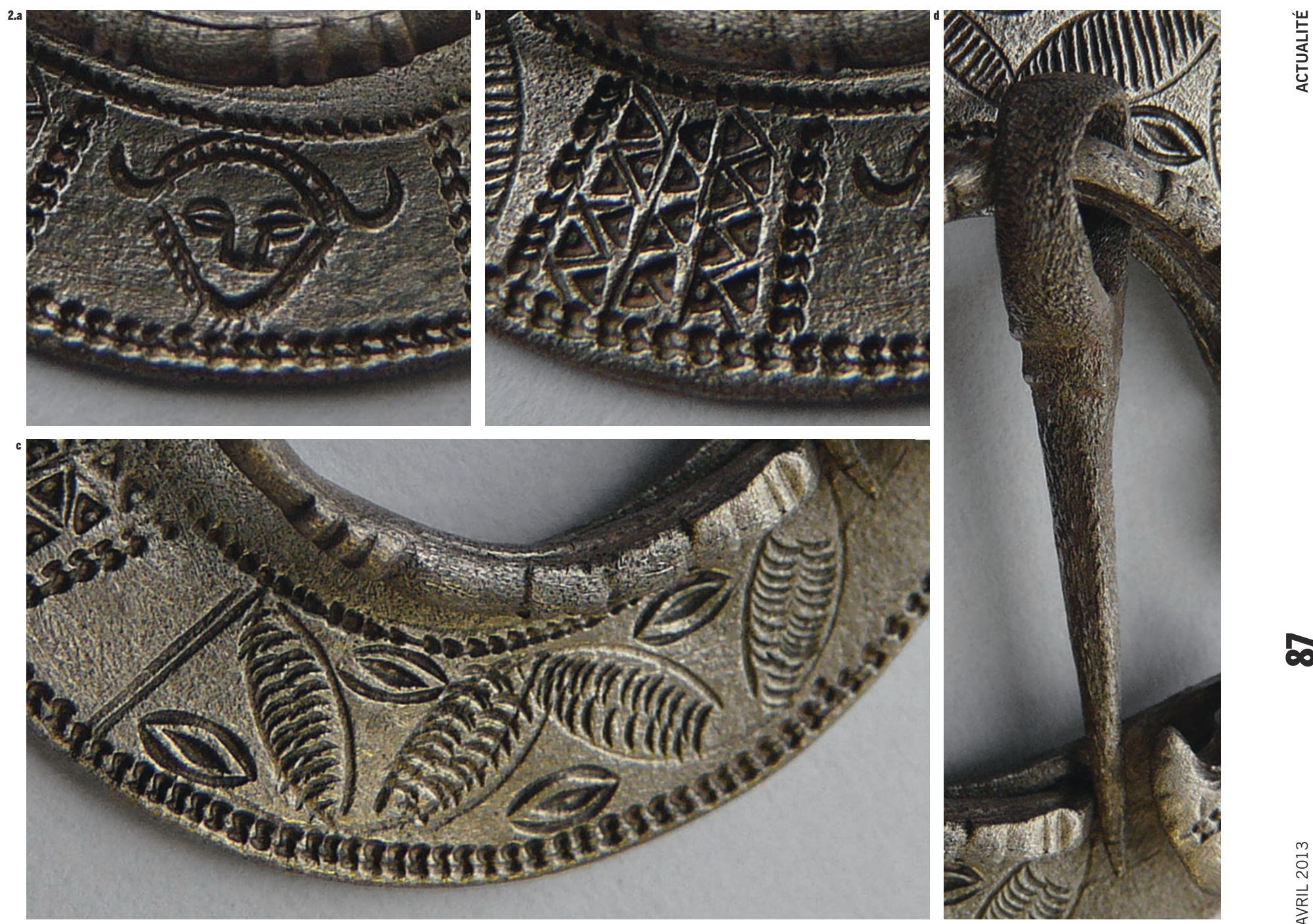

\section{Détail des différentes}

zones décorées.

Les deux extrémités de la plaque de l'oméga sont repliées sur le dessus pour former deux pattes. Grâce à l'eśpace laissé entre la plaque et le repli, ces dernières servent de blocage à l'ardillon. Elles ont un pourtour découpé et des gravures leur donnent l'allure de têtes d'animaux stylisées.

Deux petites perforations circulaires, placées à l'aplomb de ces têtes, servent à la fixation de l'anneau qui suit la courbure du vide central. La décoration est plus sobre à ce niveau avec des incisions perpendiculaires, placées par groupes de trois ou quatre en alternance avec un espace régulier ou rapproché. L'anneau cache en partie une petite ligne de $\mathrm{S}$ limitant le décor de la plaque vers le centre de l'objet.

Enfin, l'ardillon, constitué d'une longue épingle effilée repliée en boucle autour de l'anneau central, montre deux petites protubérances circulaires évoquant une tête d'oiseau à long bec de type échassier [ill. 2.d].

Cette fibule décorée trouve des parallèles en Angleterre, à Alfriston, Bishopstone, Mitcham, Orpington et Portway, et un autre exemplaire en France à Amiens, permettant d'en attribuer la production à un groupe reconnu dans le secteur du Jutland (Danemark) ou de la Scandinavie - tout au moins d'un point de vue stylistique - et caractéristique du Quoit Brooch Style. Ces productions sont fréquentes au milieu du $v^{e}$ siècle. Si sa découverte ne permet pas d'allouer une origine anglo-saxonne à son propriétaire, elle fournit une nouvelle occurrence de ce type d'objet rare et témoigne au moins des liens de ce personnage avec des produits manufacturés outreManche, à l'image des relations déjà perçues en 2006 sur une nécropole du Morbihan (Simon, 2010).

\section{Références bibliographiques}

BOUSQUET J 1971, «Informations archéologiques, circonscription de Bretagne, Ille-et-Vilaine, Rennes », Gallia, 29, p. 235-236.

LABAUNE-IEAN F., 2012, «L'étude de la céramique et des autres mobiliers ", in PouILLE D. DIR., Rennes (Illeet-Vilaine). Hôtel-Dieu, rue de la Cochardière. Les vestiges antiques de l'Hôtel-Dieu, Rapport de diagnostic, Inrap-SRA Bretagne, p. 81-95.

POULLED, 2001, Rennes, Parc des Tanneurs, document final de synthèse, diagnostic, Afan-SRA Bretagne.

SimON L., 2010, « Parures métalliques Quoit Brooch Style ", Archéopages, 29, avril 2010, p. 88-89.

SUZUKI S., 2000, The Quoit Brooch Style and anglo-saxon Settlement. A casting and recasting of cultural identity symbols, Woodbridge (GB) Rochester NY (USA), the Boydell Press, 218 p. 